\title{
Generation of high dynamic range for enhancing the panorama environment
}

\author{
Roa'a M. Al_airaji ${ }^{1}$, Ibtisam A. Aljazaery ${ }^{2}$, Suha Kamal Al_Dulaimi ${ }^{3}$, Haider Th. Salim ALRikabi ${ }^{4}$ \\ ${ }^{1}$ Science College, Babylon University, Iraq \\ ${ }^{2}$ Engineerning College, Babylon University, Iraq \\ ${ }^{3}$ Agriculture College, AL-Qasim Green University, Iraq \\ ${ }^{4}$ Electrical Engineering Dept.College of Engineering Wasit University, Iraq
}

\begin{tabular}{l}
\hline \hline Article Info \\
\hline Article history: \\
Received Mar 11, 2020 \\
Revised May 24, 2020 \\
Accepted Jun 3, 2020 \\
\hline
\end{tabular}

Keywords:

BBF search algorithm High dynamic range (HDR)

Image stitching

Inverted local patterns

Panorama

SIFT features

\begin{abstract}
This paper presents a methodology for enhancement of panorama images environment by calculating high dynamic range. Panorama is constructing by merge of several photographs that are capturing by traditional cameras at different exposure times. Traditional cameras usually have much lower dynamic range compared to the high dynamic range in the real panorama environment, where the images are captured with traditional cameras will have regions that are too bright or too dark. A more details will be visible in bright regions with a lower exposure time and more details will be visible in dark regions with a higher exposure time. Since the details in both bright and dark regions cannot preserve in the images that are creating using traditional cameras, the proposed system have to calculate one using the images that traditional camera can actually produce. The proposed systems start by get LDR panorama image from multiple LDR images using SIFT features technology and then convert this LDR panorama image to the HDR panorama image using inverted local patterns. The results in this paper explained that the HDR panorama images that resulting from the proposed method is more realistic image and appears as it is a real panorama environment.
\end{abstract}

This is an open access article under the CC BY-SA license.

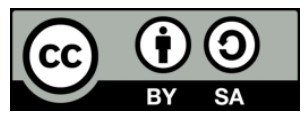

Corresponding Author:

Roa'a M. Al_airaji,

Science College,

Babylon University,

Iraq.

Email: roaaalairaji90@gmail.com

\section{INTRODUCTION}

The real panorama environment usually has a much higher dynamic range between the smallest luminance densities and the highest luminance densities than what traditional cameras can capture and what monitors can produce, since they usually work in low dynamic range (LDR). This causes to images that displayed on monitors and captured with cameras to have regions that are too bright or too dark or smaller contrast. However, for some applications, such as immersive panoramas and photorealistic superimposition of objects using image-based lighting, it is necessary to know the high dynamic range (HDR) values in the scene in order to get persuasive results $[1,2]$.

Because of numerous cameras operate with low dynamic range, all pixels above or below specific brightness value will be either white or black. According to the exposure time, the camera is set to the pixels will be clamped to white or black. If the image taken with high exposure time, the details in dark regions will be preserving while bright regions will be white. Conversely, if the image taken with low exposure time, the details in bright regions will be preserving while the dark regions will be nearly black [3]. 
Variations in environment illumination meant that must be adjusting the times of exposure according to the surrounding conditions. In other word, the exposure time must be extended when the cameras is operates in dark conditions to avoid negative saturation. On the contrary, the exposure time must be shortening when the camera is operating in bright conditions to avert positive saturation. However, the negative and positive saturation exist within the single image when it contains both dark and bright regions, the visual quality will be degrade because the details cannot be rendering precisely in the exceedingly bright and dark regions. The images which preserve the details in both the bright and the dark regions are want to achieve, such images have higher dynamic range and are called HDR images. High dynamic range algorithms are using to expand the dynamic range of the image to rendering the details in the dark and bright regions in a same image [4, 5]. There are several methods are used for creating a HDR image. In $[6,7]$, the first method is developed to get HDR image by capturing the same LDR image in three different exposure times. then using gradient based synthesis to merging these image, the three images are compared based on Gaussian blend and cut function to calculate the pixels in the HDR image with the goal of preserve the regions with higher quality from each image, the three images are then merged to compose one HDR image.

Humblot-Renaux proposed method to acquisition the image of a finger vein includes of captures an image at multi exposure-time and fusion these exposures into one HDR image to get the details of vein. The research proposed two HDR methods: weighting with a moving mean filter and basic scalar weighting [8]. Unluckily, the method of multi exposure time takes high computational costs and long processing time. Vonikakis et al. reducing this using single exposure algorithm that consists of three phases: linear stretching, estimation of block parameter, and modification of contrast, HDR image can produce rapidly using this method but the image resolution may be degrade by the down- and up-sample [9, 10]. Guarnieri et al. [11] used exponential and logarithms-based edge preserve low-pass and multiple resolution accelerate. Unluckily, using the operations of iterative inevitably, the time of computation is increases. This paper proposed algorithm to get HDR image using single image and one pass processing flow to speed up the process of computation, where the proposed system in this paper consist of two main phase, the first phase is stitching of multiple images to get the panorama and the second phase is convert the panorama image to HDR panorama image.

\section{RESEARCH METHOD}

The proposed system consists of two main phases, each of which consists of others sub-phase as described in Figure 1. The first phase is LDR panoramic image generation. That consists of two phases: image registration and blending. The second phase is Convert LDR to HDR panoramic image.

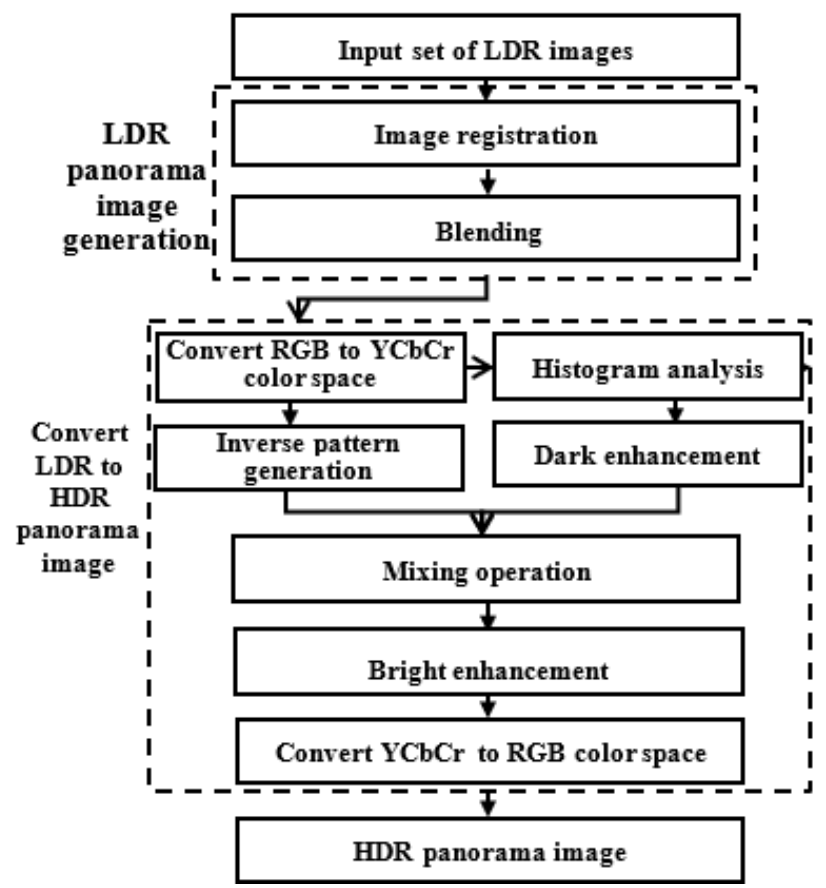

Figure 1. Block diagram of the proposed method 


\subsection{LDR panorama image generation}

Panorama generation or (image stitching) is a process of combining more than one photographic image with overlapped regions to create a panoramic view. With the number of images that are stitching, the amounts of information are increases and the view is enlarging. Panorama generation phase consist of two phases as follow:

\subsubsection{Image registration}

This phase consists of four sub-phases: features detection and description, features matching, homographs, and global alignment as follow:

a. SIFT feature extraction

SIFT features provide a group of key points of an input images that are not affect by the complexities that the other methods are experiencing, such as rotation and scaling. The key points are defined as the minima and maxima results of the different of Gaussian (DOG) function applying in scale space to the input images. The SIFT algorithm applies four steps to extract of these features as follow [9, 12-14]:

- Scale-space extrema detection

The key points are interest point, different of Gaussian (DOG) function is use to achieve this effectively by convolving the images with filters of Gaussian at different scales. The visibility of the edges in the images is increases using the DOG function. The scale space function for the image is defining as:

$$
L(x, y, \sigma)=G(x, y, \sigma) * I(x, y)
$$

where $\mathrm{G}(\mathrm{x}, \mathrm{y}, \sigma)$ is a variable scale Gaussian, $\sigma$ is scale value, $\mathrm{I}(\mathrm{x}, \mathrm{y})$ is the input image, and $*$ is the convolution operator. The stable key points positions in the scale-space are detecting using difference of Gaussians (DOG), scale-space extrema $\mathrm{D}(\mathrm{x}, \mathrm{y}, \sigma)$ is locating by calculate the differences among two images, one with scale $\mathrm{k}$ times the other as shown in (2):

$$
D(x, y, \sigma)=L(x, y, k \sigma)-L(x, y, \sigma)[15]
$$

The local minima and maxima of $\mathrm{D}(\mathrm{x}, \mathrm{y}, \boldsymbol{\sigma})$ is detecting by comparing each point with its nine neighbors down and up one scale and its eight neighbors at the same scale. The point is an extrema, if it is maximum or minimum of overall these points $[16,17]$.

- Key point Llocalization

More number of key points is generating in stage of the scale-space extrema detection, this stage attempts to reduce these points by finding those that are poorly localizing on an edge or have low contrast. This is doing based on Laplacian value that computing for each key point found in previous phase. The location of extrema is computed by:

$$
\mathrm{Z}=-\frac{\partial^{2} \mathrm{D}^{1}}{\partial \mathrm{x}^{2}} \frac{\partial \mathrm{D}}{\partial \mathrm{x}} \quad[18]
$$

The point is excluding, if the function value at $\mathrm{z}$ is below a threshold value [12].

- Orientation assignment

In this step, the consistent orientation to the key points is assigning based on local features of the image. The descriptors of the key points can then be representing relative to this orientation, hence, the invariance to image rotation can be achieves. The method to find the orientation is start by using the key point scale to determine the Gaussian smooth image L, from above and calculate gradient magnitudes and orientations $\theta$. After that, from the gradient orientations of the key points, an orientation histogram is calculated. Lastly, the orientation to the key points can be assigned according to the histogram [19, 20].

- Key point descriptor

The key point descriptors are created using gradient information, used above. Rotate information of the gradient to line up with the key point orientation and then weighting by a Gaussian. After that, this data is using to creating a group of histograms over a windows centering on the key point. Key point descriptors usually use a group of 16 histograms, ranged in a $4 \times 4$ grid, each with 8 orientation bins [16, 20, 21].

b. Features matching

Feature points matching can be imputed to a problem of similarity search by distance function in high dimensional space. This paper uses best bin first (BBF) algorithm for matching the feature vectors that generated in previous step. The BBF contains of six parts in data structure: the right and the left tree pointer. The value $\mathrm{n}$ represents the number of descript in this $\mathrm{k}-\mathrm{d}$ tree. The division value $\mathrm{v}$ where it is containing 
the median value of each feature descriptor in dimension i. the discriminator/for divide each feature descriptor, which mark the dimension in which feature descriptor exhibit the largest variance. The pointer to the list feature descriptors $[16,22]$.

The BBF also contains a priority queue and the pointer q that represent the query point. The priority queue contains two part of information: the query point (q) distance from the k-d tree node and the position of current tree. During search of nearest neighbors in BBF algorithm, an entry is a pushed to the priority queue when judgment is making at an internal node to branching in one direction. Whenever an element is an inserted, the priority queue will be sorting ascending by the distances. After examine the leaf node, the top entry in the priority queue is removing and the next search is beginning from the sub-tree that contains the next feature descriptor. The search is continuing until the queue is empty of the query number exceed qmax (qmax represent maximum query number in this paper [23-25].

c. Homography using RANSAC

In this phase, the useful information that obtained from features matching phase is use to do image matching. In image matching phase, the aim is found the correctly feature matching and determine which image is neighbor to another image. The method in this paper use RANSAC to calculate a set of inliers that are convenient with a homographic among the images. RANSAC is a repeated algorithm use to calculate parameters of a statistical model from set of observzed data that contain outliner. Where it is start by randomly selecting four pairs of feature points. Then, calculate homography $(\mathrm{H})$. After that, calculate inliers and save it where SSD $\left(\mathrm{p}_{\mathrm{i}}^{\prime} \mathrm{H} \mathrm{p}_{\mathrm{i}}\right)<\mathrm{thr}$. Lastly, use inliers to re-calculate least squares $\mathrm{H}$ if the inliers grow over a specific threshold [26-30].

After the RANSAC phase, the homography $\mathrm{H}_{\mathrm{ij}}$ among every matching image is calculating. For example, image $\mathrm{i}$ and image $\mathrm{j}$, to stitching the images, determine one image in randomly manner as start image a and all points in other image (i.e. $\mathrm{x}_{\mathrm{j}}$ ) are projecting to the coordinates of the image a. the new location of the new points of every neighbor image $\mathrm{j}$ of a is calculating using (4):

$$
x_{a}=H_{a j} x_{j}
$$

the series of homography matrices, i.e. $\mathrm{x}_{\mathrm{a}}=\mathrm{H}_{\mathrm{am}} \mathrm{H}_{\mathrm{mn}} \ldots \mathrm{H}_{\mathrm{hi}} \mathrm{x}_{\mathrm{i}}$ are used to find a sequence of linked images transform the image [26].

d. Global alignment

The bundle adjustment is the most relevant method that is merges multi images of the same scene into a precise $3 \mathrm{D}$ reconstruction. The objective is to obtain set of alignment parameters that are global consistency that reduce the poor registration among each pair of images. Initial estimate of the 3D position of feature in the image must be compute, in addition to estimate of the positions of the camera. Then, applying the bundle adjustments to computing the optimal values for camera positions and the 3D reconstruction of the scene, by minimize the log-likelihood of the inclusive feature projection mistakes using the algorithm of least squares. In order to do this, require to expand the criteria of pairwise match to a globally energy function that includes all of the per image pose parameter. After compute the global alignments, the local adjustment is performing such as parallax elimination to minimize blur and dual image that occur to local mis-registration $[31,32]$.

\subsubsection{Blending}

Image blending is a technique used to remove the joint between two consecutive images and make the transition between each two consecutive images smoother. In other word, Image blending used in image stitching to handle overlapping regions and produce an attractive panorama. There are many image-blending methods, such as Image Pyramid blending, gradient domain and alpha (feathering) image blending that used in this paper. In which, the feathering image blending method takes the average of pixel values in the blending region from the two overlapped images [26, 33, 34].

\subsection{Convert LDR to HDR panoramic image}

After generate LDR panorama, the second phase is converting convert LDR to HDR panoramic image. Where the color space of the LDR panorama is convert from RGB to YCbCr. To compute of HDR, inverse kernel based on block processing in the $\mathrm{Y}$ channel is applying to generate the inverse pattern $\mathrm{y}_{\text {inv. }}$. On the other path, the histogram analysis used to classify the image type as bright, extreme, and dark type based on luminance signal Y. after that, enhance the regions with low grey level for extreme and dark type by applying the dark enhancement. Then, the result of the dark enhancement is combined with result of the inverse pattern, and the next step, the bright enhancement, is performed using the brightness control variable k. Lastly, the HDR color is generating by convert from YCbCr to RGB. 


\subsubsection{Inverse pattern generation}

The inverse kernel consists of three steps:

a. Maximum fill

This step is necessary to preserve the information of the thinning lines and to overcome the distortions. The method start by divide the image into blocks each with $2 \times 2$ size ( 4 pixels in each block). Then, each four pixels in specific block are filling by the maximum grey level value of the four pixels belongs to that block as explained in Figure 2.

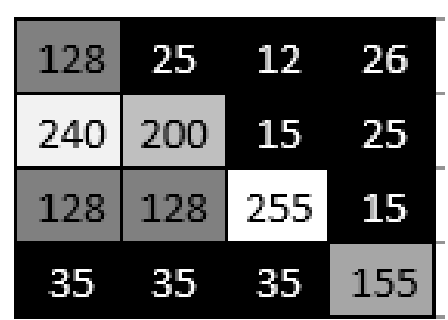

(a)

\begin{tabular}{|c|c|c|c|}
\hline 240 & 240 & 26 & 26 \\
\cline { 1 - 2 } 240 & 240 & 26 & 26 \\
\hline 128 & 128 & 255 & 255 \\
\hline 128 & 128 & 255 & 255 \\
\hline
\end{tabular}

(b)

Figure 2. Images block, (a) Original image, (b) Maximum fill results

b. Low pass filter

The jagged edges that resulting from the maximum fill step need to be smoother and the low pass filter achieve this as follow:

$$
Y_{l p f}(i, j)=\frac{\sum_{k=-1}^{1} \sum_{l=-1}^{1} Y_{\max }(i+k, j+l)}{9}
$$

where $Y_{\max }(\mathrm{i}, \mathrm{j})$ represent pixel value at coordinate $(\mathrm{i}, \mathrm{j})$ in the image that result from the maximum fill. c. Inverse function

Inverse function is used to avoid positive and negative saturations by darken bright regions and lighten dark regions. To improve performance in the dark regions, the squares linear function to this as follow:

$$
Y_{i n v}=\frac{\left(255-Y_{l p f}(i, j)\right)^{2}}{255}
$$

\subsubsection{Histogram analysis}

The histogram is used to determine the type of image by analysis of the luminance signal Y. The image is categorized into one of three categories Based on the distribution of light in histogram: Bright type, most of the gray levels in the image are high. Dark type, most of the gray levels in the image are low. Extreme type, most of the gray levels in the image are either low or high. The two parameters, Lo and Hi are used to do this as follows:

$$
\begin{aligned}
& L_{0}=\sum_{i=0}^{t h 1}(h(i) \times 2)+\sum_{i=t h 1}^{t h 2} h(i) \\
& H i=\sum_{i=t h 3}^{t h 4} h(i)+\sum_{i=t h 4}^{255}(h(i) \times 2)
\end{aligned}
$$

where $\mathrm{h}$ (i) represent the $i$ th histogram level. Lo represent summation the double number of low gray level range from zero to Th1 (the factor is multiplying by (2) among zero to Th1 to extra dark feature enhancement), and the number of low gray level range from Th1 to Th2. Hi represent summation the number of high gray level range from Th3 to Th4, and the double number of high gray level range from Th4 to 255 (the factor is multiplying by (2) among Th4 to 255 to extra bright feature enhancement). The values of thresholds are (Th1=20, Th2=55, Th3=200, and Th4=235). The image is categorized as dark type $\mathrm{f} \mathrm{Lo}>2 \mathrm{Hi}$. The image is categorized as bright type if $2 \mathrm{Lo}<\mathrm{Hi}$. The image is categorized as extreme type if $2 \geq(\mathrm{Hi} / \mathrm{Lo}) \geq 1 / 2$ and $\mathrm{Lo}$ and $\mathrm{Hi}>(\mathrm{N} / 4)$, where the $\mathrm{N}$ represent the number of pixels. 


\subsubsection{Dark enhancement}

The dark pixels in extreme type image and dark type image are lightening using (9):

$$
Y_{\text {dark }}(i, j)= \begin{cases}y_{\text {in }}(i, j)+\left(T h_{\text {dark }}-y_{\text {in }}(i, j)\right) \times 0.05 & \text { if } y_{\text {in }}<T h_{\text {dark }} \\ y_{\text {in }}(i, j) & \text { if } y_{\text {in }} \geq T h_{\text {dark }}\end{cases}
$$

where $\mathrm{y}_{\mathrm{in}}$ represents the original input $\mathrm{Y}$ signal, $\mathrm{Th}_{\mathrm{dark}}$ is threshold value and the value of threshold based on the image type. The operation involves reduce the number of pixels with low grey levels in order to overcome of the negative saturation. When the image type is bright, no lighting is performed and $\mathrm{Th}_{\text {dark }}=0$. When the image type is extreme, the grey level is enhancing for each pixel smaller than 50 and $\mathrm{Th}_{\text {dark }}=50$. When the image type is dark, the grey level is enhancing for each pixel smaller than 128 and $\mathrm{Th}_{\mathrm{dark}}=128$.

\subsubsection{Mixing operation}

The inverted pixel from (6) and enhancing pixel from (9) are mixing using (10):

$$
Y_{\text {mix }}(i, j)=y_{i n v} \times y_{\text {dark }} \times k
$$

where $\mathrm{k}$ is a key parameter that identified the HDR image and its value depend on the image features. For an image with eight bits grey level, the $Y_{\text {mix }}$ value is bounded at 255 as maximum value. The HDR effect will become more obvious by increasing the $\mathrm{k}$ value. Because of the inverse backlight $\mathrm{y}_{i n v}$ value of dark region is high, the dark pixels are made brighter. In the opposite case, Because of the low $y_{\text {inn }}$, the brighter pixels are making dark to prevent pixels from become over-exposer.

To handle each type of image, the adaptive $\mathrm{k}$ parameter is devising and the linear proportional relation among Lo and $\mathrm{k}$ is shown in the following equation:

$$
k=m \times L o+b
$$

where $(m)$ represents the linear slope, $\mathbf{b}$ is an offset. The linear variation is reducing, when the slope is small. The slop is an attained using the following equation:

$$
m=\tan \theta=\frac{k_{2}-k_{1}}{L o_{2}-L o_{1}}
$$

The estimating Lo value is high, when the image type is dark; therefore, need to increase the parameter $\mathrm{k}$ to enhancement the brightness. Offset value $\mathrm{b}$ can be computes using the following equation:

$$
b=k_{1}-m \times L o_{1}
$$

After the operation of mixing, the luminance of the bright regions is compressed and the dark regions are increased. The grey level is an increasing to expand the dynamic range using the following equation:

$$
Y_{H D R}(i, j)= \begin{cases}y_{\text {mix }}(i, j)+\left(y_{\text {dark }}(i, j)-T h_{b}\right)^{2} \times 0.005 & \text { if } y_{\text {dark }}(i, j) \geq T h_{b} \\ y_{\text {mix }}(i, j) & \text { if } y_{\text {dark }}(i, j)<T h_{b}\end{cases}
$$

After that, the luminance ranges from 0 to 255 is expanded using AGC. Finally, the $\mathrm{YCbCr}$ color space is converting to the RGB color space.

\section{RESULTS AND DISCUSSION}

The results are making to analyze the quality of HDR panorama that produced with the proposed system in this paper. The results explained that the HDR have more advantages over the LDR panorama. The results in Figure 3 explained HDR panorama more realistic from LDR panorama for outdoor environment. Since an outdoor environment has extremely high dynamic range, the HDR that it is colorful necessary to make the user feeling immersive and realistic. 

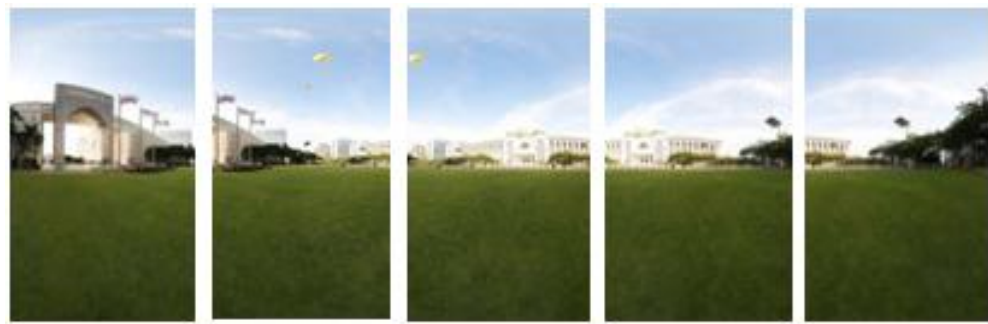

(a)

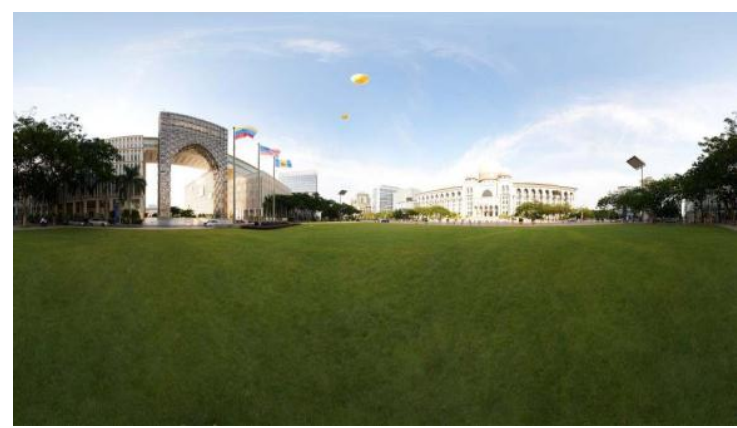

(b)

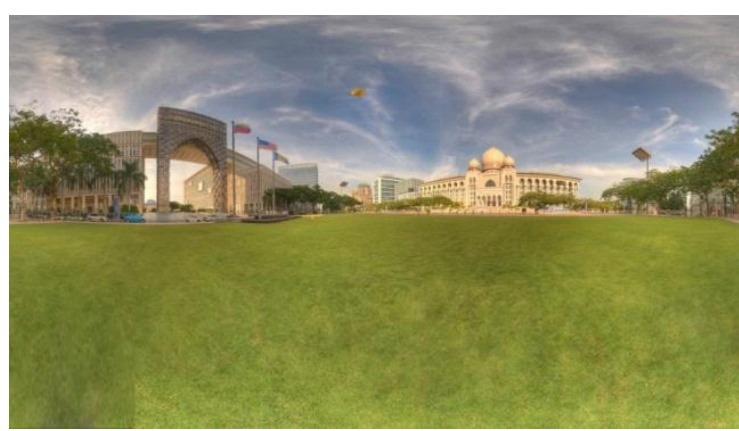

(c)

Figure 3. Captured outdoor image, (a) Set of input LDR images, (b) LDR panorama, (c) HDR panorama

The results in Figure 4 explained some details on the LDR panorama are lost. Objects that are invisible on the LDR picture but it is visible on the HDR panorama. Bright regains are overexposing and objects that are not obvious on the LDR panorama picture but it is obvious on the HDR one. The results in Figure 5 explained the difference between the LDR and the HDR panorama is not very distinctive on the first sight but when enlarge the figure additional details can be noticed on the HDR panorama image.
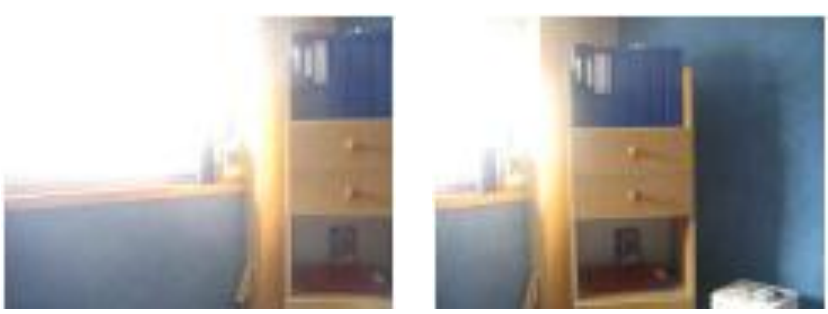

(a)

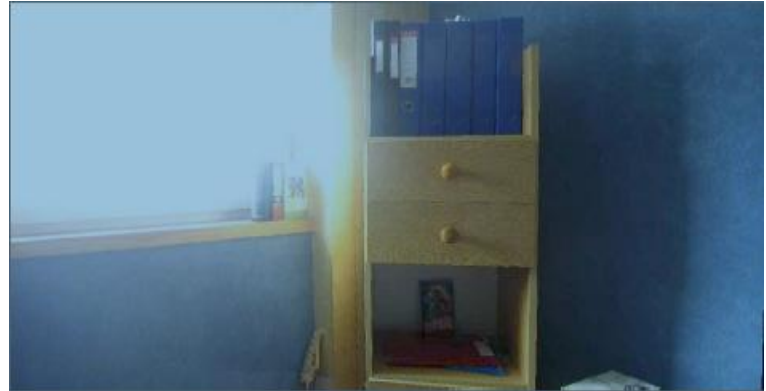

(b)

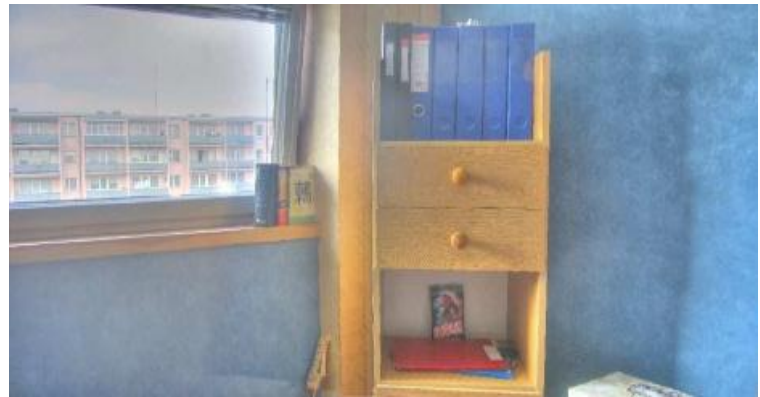

(c)

Figure 4. Captured indoor panorama, (a) Set of input LDR images, (b) LDR panorama, (c) HDR panorama 

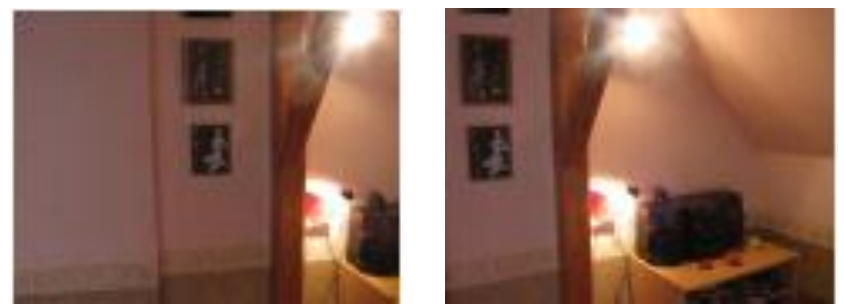

(a)

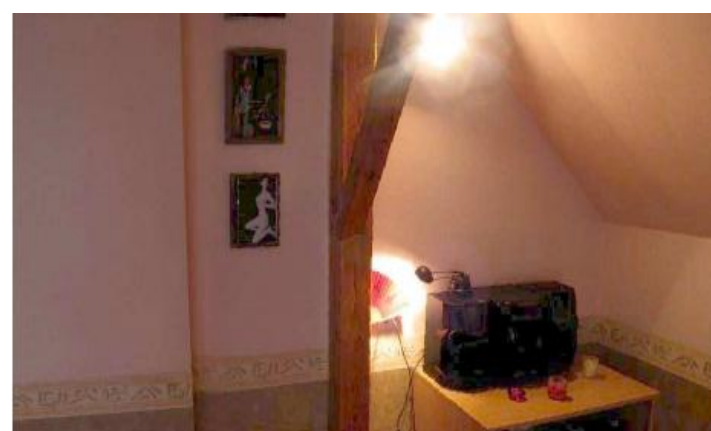

(b)

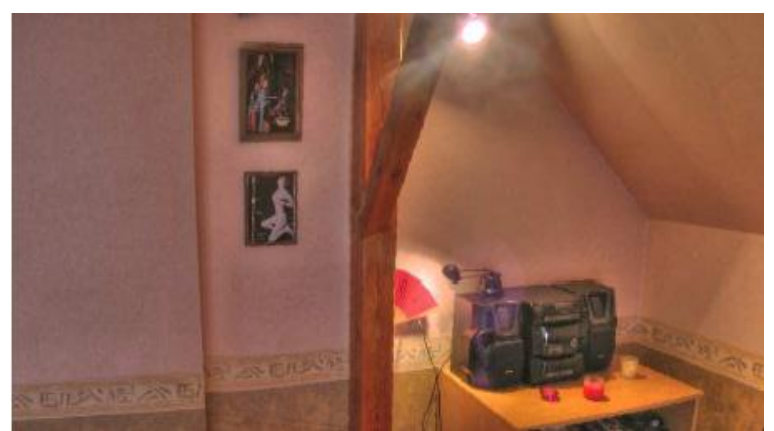

(c)

Figure 5. Captured indoor panorama, (a) Set of input LDR images, (b) LDR panorama, (c) HDR panorama

\section{CONCLUSION}

This paper presents a high performance HDR panorama method. The results explained that the proposed method is able to producing high quality HDR panorama by adjust over exposed and under exposed regions. This issue is necessary in the panorama image, since the luminance can vary significantly in the two images. By convert LDR to HDR panorama, one can process those lightning variance ties. The producing HDR panorama appears natural (real scene), rather than unreal. The proposed method for HDR panorama not uses recursive process instead it uses single pass process, where this help to minimize computation cost, make it appropriate in real time processing.

\section{REFERENCES}

[1] F. Okura, M. Kanbara, and N. J. V. R. Yokoya, “Aerial full spherical HDR imaging and display,” Virtual Reality, vol. 18, no. 4, pp. 255-269, 2014.

[2] J. Zhang, K. Sunkavalli, Y. Hold-Geoffroy, S. Hadap, J. Eisenman, and J-F. Lalonde, "All-weather deep outdoor lighting estimation," in Proceedings of the IEEE Conference on Computer Vision and Pattern Recognition, pp. 10158-10166, 2019

[3] L. M. Leo and A. J. Simla, "2D to 3D conversion of dental images using deep neural network," Journal of Chemical and Pharmaceutical Sciences, vol. 10, no. 3, pp 1432-1436, 2017.

[4] J. Unger, J. Kronander, P. Larsson, S. Gustavson, J. Löw, A. Ynnerman, "Spatially varying image based lighting using HDR-video," Computers \& Graphics, vol. 37, no. 7, pp. 923-934, 2013.

[5] H. TH. Alrikabi, A. H. M. Alaidi, A. S. Abdalrada, and F. T. Abed, "Analysis the efficient energy prediction for 5g wireless communication technologies," International Journal of Emerging Technologies in Learning, vol. 14, no. 8, pp. 23-37, 2019.

[6] S. Mann, "Compositing multiple pictures of the same scene," Proc. IS\&T Annual Meeting, pp. 50-52, 1993.

[7] R. J. Van Der Vleuten, J. H. C. Stessen, and J. G. R. Van Mourik, "Methods and apparatuses for creating code mapping functions for encoding an HDR image, and methods and apparatuses for use of such encoded images," U.S. Patent No. 10,194,162, 2019.

[8] G. Humblot-Renaux, "Implementation of HDR for image acquisition on a finger vein scanner," Thesis, Electrical Engineering, Mathematics and Computer Science, University of Twente, 2018.

[9] I. A. Aljazaery, "Encryption of images and signals using wavelet transform and permutation algorithm," Journal of University of Babylon, vol. 22, no. 9, pp. 2617-2634, 2014.

[10] X. Wang, Q. Jiang, F. Shao, K. Gu, G. Zhai, and X. Yang, "Exploiting local degradation characteristics and global statistical properties for blind quality assessment of tone-mapped HDR images," IEEE Transaction on Multimedia, p. $1,2020$.

[11] G. Guarnieri, S. Marsi, and G. Ramponi, "High dynamic range image display with halo and clipping prevention," IEEE Transactions on Image Processing, vol. 20, no. 5, pp. 1351-1362, May 2011. 
[12] D. A. Delphin, M. R. Bhatt, D. Thiripurasundari, and I. Sciences, "Holoentropy measures for image stitching of scenes acquired under CAMERA unknown or arbitrary positions," Journal of King Saud University - Computer and Information Sciences, 2018.

[13] M. Oktiana, F. Arnia, Y. Away, and K. Munadi, "Features for cross spectral image matching: a survey," Bulletin of Electrical Engineering and Informatics, vol. 7, no. 4, pp. 552-560, 2018.

[14] M. A. Rasyidi and T. Bariyah, "Batik pattern recognition using convolutional neural network," Bulletin of Electrical Engineering and Informatics, vol. 9, no. 4, pp. 1430-1437, 2020.

[15] X. Hu, Y. Tang, and Z. Zhang," Video object matching based on SIFT algorithm," International Conference on Neural Networks and Signal Processing, IEEE, pp. 412-415, 2008.

[16] A. Zhou and W. Shao, "Surface defect analysis of rotary metal parts," 2016 IEEE International Conference on Mechatronics and Automation, pp. 149-153, 2016.

[17] H. T. S. ALRikabi, "Enhancement of the MIMO-OFDM Technologies," International Journal of Scientific Engineering and Research, vol. 4, no. 8, pp.20-25,2013.

[18] G. Clemente and M. D'Elia, "Spectrum of the Laplace-Beltrami operator and the phase structure of causal dynamical triangulations," Physical Review, vol. 97, no. 12, p. 124022, 2018.

[19] E. Cardoso, H. A. Risch, L. P. Laheras, V. Luiz, P. S. Rodrigues and G. A. Wachs-Lopes, "Image stitching using non-extensive statistics," 2019 XV Workshop de Visão Computacional (WVC), Spp. 84-88, 2019.

[20] R. H. Doshi, R. A. Soni, B. Agrawal, and R. L. Naik, "Study, survey and analysis for media selection,"Bulletin of Electrical Engineering and Informatics, vol. 1, no. 1, pp. 1-6, 2012.

[21] O. H. Yahya, H. Th. S. AlRikabi, I. A. Aljazaery, "Reducing the data rate in internet of things applications by using wireless sensor network," International Journal of Online and Biomedical Engineering, vol. 16, no. 3, pp. 107-116, 2020.

[22] R. Li, L. Xing, S. Napel, and D. L. Rubin, "Radiomics and radiogenomics: Technical basis and clinical applications," Chapman and Hall/CRC, 2019.

[23] H. Liu, M. Deng and C. Xiao, "An improved best bin first algorithm for fast image registration," Proc. of 2011 Int. Conf. on Electronic \& Mechanical Engineering and Information Technology, pp. 355-358, 2011.

[24] S. Li, G. Cheng, and C. Li, "Relaxing relationship queries on graph data," Journal of Web Semantics, vol. 61-62, March 2020.

[25] H. Santoso, A. Harjoko, and A. E. Putra, "Efficient K-nearest neighbor searches for multiple-face recognition in the classroom based on three levels DWT-PCA," vol. 8, no. 11, pp. 112-122, 2017.

[26] E. Adel, M. Elmogy, and H. Elbakry, "Image stitching based on feature extraction techniques: A survey," International Journal of Computer Applications, vol. 99, no. 6, pp. 1-8, 2014.

[27] I. A. Aljazaery, A. A. Ali, and H. M. Abdulridha, "Classification of electroencephalograph (EEG) signals using quantum neural network," Signal Processing: An International Journal, vol. 4, no. 6, pp. 329-337, 2011.

[28] I. A. Aljazaery, H. T. S. Alrikabi, and M. R. Aziz, "Combination of hiding and encryption for data security," International Journal of Interactive Mobile Technologies, vol. 14, no. 9, pp. 34-47, 2020.

[29] J. Zhang, C. Wang, S. Liu, L. Jia, J. Wang, and J. JZhou, "Content-aware unsupervised deep homography estimation," arXiv preprint arXiv:1909.05983,2019.

[30] S. S. N. Bhuiyan and O. O. Khalifa, "Efficient 3D stereo vision stabilization for multi-camera viewpoints," Bulletin of Electrical Engineering and Informatics, vol. 8, no. 3, pp. 882-889, 2019.

[31] V-D. Hoang, D-P. Tran, N. G. Nhu, and V-H. Pham, "Deep feature extraction for panoramic image stitching," in Asian Conference on Intelligent Information and Database Systems, Springer, pp. 141-1512020.

[32] J. Feng, C. Ai, Z. An, Z. Zhou, and Y. Shi, "A feature detection and matching algorithm based on Harris Algorithm," International Conference on Communications, Information System and Computer Engineering (CISCE), pp. 616-621, 2019.

[33] H. Pourghassem and S. Daneshvar, "A framework for medical image retrieval using merging-based classification with dependency probability-based relevance feedback," Turkish Journal of Electrical Engineering \& Computer Sciences, vol. 21, no. 3, pp. 882-896, 2013.

[34] Z-B. Pan and X-L. Hu, "Research and design of MCU interface based on FPGA real-time blending image system," Techniques of Automation and Applications, vol. 1, 2011.

\section{BIOGRAPHIES OF AUTHORS}

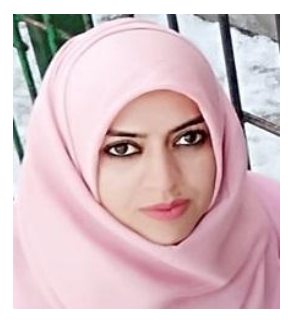

Roa'a M. Al_airaji received the Bachelor degree from Department of Computer, College of Science, University of Babylon, in 2012. Received the Master degree from Department of software, College of Information Technology, University of Babylon, in 2018. Currently work as assistant teacher in College of Science, University of Babylon. 

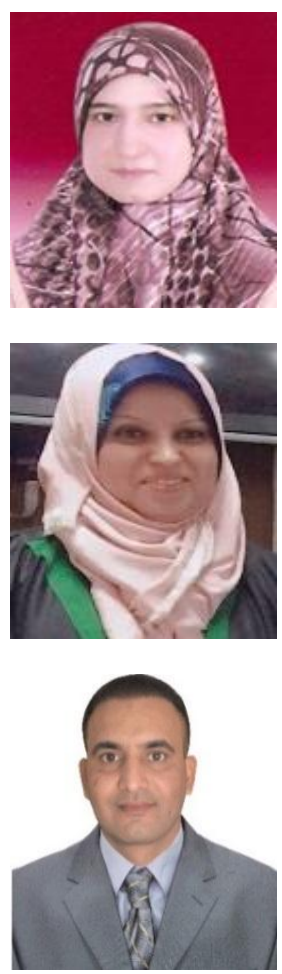

Ibtisam A. Aljazaery received the Bachelor degree from University of Technology/Iraq in computer and cotrol engineering department. Received the Master degree from College of Engineering/ computer and cotrol engineering department, University of Basrah/Iraq. Currently work as a teacher in College of Engineering, University of Babylon.

Suha Kamal Al_Dulaimi received the Bachelor degree from Department of Computer, College of Science, University of Babylon. Received the Master degree from Department of software, College of Information Technology, University of Babylon, in 2018. Currently work as assistant teacher in College of Agriculture, University of AL-Qasim Green.

Haider Th. Salim ALRikabi received his B.Sc. degree in Electrical Engineering in 2006 from the Al Mustansiriya University in Baghdad, Iraq. his M.Sc. degree in Electrical Engineering focusing on Communications Systems from California state university/Fullerton, USA in 2014. $\mathrm{He}$ is currently one of the faculty member in college of engineering, electrical engineering department, Wasit University in Al Kut, Wasit, Iraq His current research interests include Communications systems with mobile generation, Control systems, intelligent technologies, smart cities, and Internet of Things (IoT). His address: Al Kut city - Hay ALRabee, Wasit, Iraq Contact: +9647732212637, E-mail: hdhiyab@uowasit.edu.iq. The number of articles in national databases -10 . The number of articles in international databases -20 